The aim of this study was to review admissions for complications secondary to VZV infection over a six month period (January - July 2017) in a Dublin paediatric hospital. The VZV vaccine is currently available in Ireland but not included on the childhood universal immunisation programme.

Method Patients who were admitted to Children's University Hospital Temple Street (CUHTS) with complications secondary to VZV infection were identified. These patients' demographics, symptoms and management were recorded and analysed.

Results Thirteen children were admitted to CUHTS between January and July 2017 as a result of complications of chickenpox infection. Five children were male. The ages ranged from 27 weeks to 7 years (median=1.5 years). All children were previously well, with no history of immunodeficiency. All patients had commenced the routine vaccination schedule but none had received the VZV vaccine.

Complications included cellulitis, osteomyelitis, septic arthritis, encephalitis, toxic shock syndrome, sepsis, and intracranial abscess. Some patients had several complications at once. The length of time from VZV infection to onset of complication ranged from no history of chickenpox (but VZV identified on CSF PCRs) to 17 days after the rash had crusted over (median $=4.5$ days). Six children (46\%) had Group A Streptococcus identified as a causative organism for their complication.

The length of hospital stay ranged from 1 day to 27 days (median=6). One child was admitted to PICU for 9 days. Two patients required surgery (orthopaedic, ENT and neurosurgery). Four patients required $>6$ weeks of antibiotics. All children have had varying levels of outpatient follow up and treatment.

Conclusion While the majority of VZV cases are mild and self-limiting, there is significant patient morbidity and healthcare costs associated with severe or complicated varicella infections. Permanent disability occurs particularly with intracranial and orthopaedic complications. There is an added economic impact for parents taking time off work to be with their sick child. We believe that universal VZV vaccination needs to be reconsidered.

\section{GP171 MICROFLORA OF THE CONJUNCTIVAL CAVITY IN NEWBORN CHILDREN BORN NATURALLY}

\begin{abstract}
1,2Maria Zaytseva*, 3,4 Irina Vorobtsova, Vladimir Brzheskiy ${ }^{3}$, Elena Spasibova 3,5, ${ }^{6}$ Nataly Bistritskaya, ${ }^{7}$ Marina Khramtsova, ${ }^{7}$ Nataly Kisel', Larisa Strukova ${ }^{7},{ }^{8}$ Maria Pavlova, ${ }^{9}$ Elizabeth Khonko, ${ }^{9,4}$ Ksenia Sokolova. ${ }^{1}$ SPb GBUZ 'Diagnostic Center No. 7' (eye) for adults and children, Saint-Peterburg, Russian Federation; ${ }^{2}$ Federal State Budget Educational Institution of Higher Education "St. Petersburg State pediatric medical university» Ministry of Health of the Russian Federation, Saint-Peterburg, Russian Federation; ${ }^{3}$ Federal State Budget Educational Institution of Higher Education "St. Petersburg State pediatric medical university» Ministry of Health of the Russian Federation, Saint-Petersburg, Russian Federation; ${ }^{4}$ St. Petersburg State Budgetary Institution of Health Care 'Maternity Hospital No. 16”, Saint-Petersburg, Russian Federation; ${ }^{5}$ Federal State Budget Scientific Institution 'Scientific Research Institute of Obstetrics, Gynecology and Reproduction named after D. Ott», Saint-Petersburg, Russian Federation; ${ }^{6}$ Federal State Budget Scientific Institution 'Scientific Research Institute of Obstetrics, Gynecology and Reproduction named after D. Ott», Saint-Petersburg, Russian Federation; ' SPb GBUZ 'Diagnostic Center No. 7' (eye) for adults and children, Saint-Petersburg, Russian Federation; ${ }^{8} \mathrm{SPb}$ GBUZ 'City Hospital 038 named. ON. Semashko', Saint-Petersburg, Russian Federation; ${ }^{9}$ Federal State Budget Educational Institution of Higher Education "St. Petersburg State pediatric medical university" Ministry of Health of the Russian Federation, Saint-Petersburg, Russian Federation
\end{abstract}

10.1136/archdischild-2019-epa.232
It is well known that obstetricians are trying to reduce the percentage of cesarean sections and related complications in recent years, so the features of pregnancy and delivery are very important for neonatal ophthalmology. The importance of the study of conjunctival cavity microflora is increasing due to improvement of the technologies of intraocular interventions which are performed in even earlier terms than in the past, and due to necessity for adequate perioperative prevention of their infectious complications. Presently neonatal ophthalmia is a serous problem as well.

Prevention of neonatal ophthalmia has been carried out since 1881. Nowadays many eye treatment schemes which are used after the birth of a child have been proposed. 1\% tetracycline ointment is usually used in Russia at present time.

The purpose of our research was to study the nature of the microflora of the conjunctival cavity in newborn children born by natural delivery, and to determine the sensitivity of the isolated microorganisms to antibacterial drugs used in pediatric ophthalmology as well.

60 naturally delivered newborns (120 eyes of 29 girls and 31 boys) were examined. The average gestational age was $38.8 \pm 1.39$ weeks. In 46 cases, the amniotic fluid was bright, in 14 - meconium colored. Staph. Epidermidis were isolated in $26.4 \%$ of cases from the cervical canal of women, lactobacilli were - in $17.15 \%$ of cases, the swab was sterile in $56.45 \%$ of cases.

Sensitivity of the extracted microflora to the antibacterial drugs used in ophthalmology (aminoglycosides, macrolides, fluoroquinolones, tetracyclines, penicillins) was determined by the disco-diffusion method.

Microflora from the conjunctival cavity was not found only in $28.33 \%$ of the cases of natural delivery. Coagulase-negative staphylococcus were detected in $35 \%$ of the cases, E. Coli in $18.3 \%$. Mixed-flora was found in $15 \%$ of the cases. The isolated microorganisms were $100 \%$ sensitive to fluoroquinolones (ciprofloxacin, levofloxacin and moxifloxacin), resistance to azithromycin was $48.7 \%$, and to tetracycline - $28.2 \%$.

Conjunctival cavity of newborn babies born naturally remains sterile only in $28.33 \%$ of the cases, regardless of the degree of microbial contamination of the mother's cervical canal.

Coagulase-negative staphylococci were found in $35 \%$ of cases with clinically healthy children, who were born naturally from healthy women. $19 \%$ of those are mecitillin-resistant strains. The isolated microflora of the conjunctival cavity of newborns is most sensitive to fluoroquinolones, ciprofloxacin and moxifloxacin, which must be taken into account in perioperative preparation when planning intraocular surgical interventions.

\section{GP172 SHOULD HEALTHCARE PROFESSIONALS ROUTINELY RECOMMEND A COMMERCIALLY AVAILABLE SLEEP MOVEMENT MONITOR FOR BABIES?}

${ }^{1}$ Dane Wanniarachige, 'Síofra Murphy, ${ }^{1}$ Alice Titiloye, ${ }^{1}$ Wuey Qi Ong, ${ }^{1}$ Yong Tze Hong, ${ }^{1}$ Mohammud Javed Domah, 'Ruth Hession, ${ }^{1}$ Charlie Murray, ${ }^{2}$ Eleanor Molloy*. 'Trinity College Dublin, Dublin, Ireland; ${ }^{2}$ Professor of Paediatrics and Child Health, Trinity Inst. of Neurosciences, Dublin, Ireland

\subsection{6/archdischild-2019-epa.233}

Background Commercially available baby vital monitors have been successfully marketed towards parents, specifically as an intervention to prevent Sudden Infant Death Syndrome 
(SIDS). The aim of this study was to determine if evidence suggests that commercially available sleep movement monitors should be routinely recommended by healthcare professionals. Methods A systematic literature review was undertaken to investigate the evidence for the efficacy of infant sleep monitors. The articles retrieved were then screened in accordance with the Preferred Reporting Items for Systematic Reviews and Meta-Analyses (PRISMA) guidelines.

Results Literature search yielded five relevant articles, a majority $(80 \%)$ relating to SIDS. Two studies showed the monitor was effective in accurately detecting cessation of breathing but could not comment on their efficacy with regards to SIDS prevention. A study of 53 infants using the Babysense monitor after an Apparent Life-Threatening Event (ALTE) found the monitor accurately detected apnoea and bradycardia when compared to the cardiorespiratory monitor 'Intellivue MP20 Junior' by Phillips. Two qualitative studies reported that such devices were appealing to mothers.

Discussion The medical effectiveness and reliability of these movement monitors is still a matter of controversy. Commercial monitors may be comparable to clinical cardiorespiratory monitors in terms of detection of apnoea and bradycardia. However, no article could conclude that sleep movement monitors are an effective method of SIDS prevention. Instead, healthcare professionals should emphasise interventions proven to reduce the risk of SIDS such as positioning infants on their back to sleep, or smoking cessation. Further limitations of the devices included a high rate of false alarms.

Conclusion The systematic review revealed that there is no evidence that commercially available sleep movement monitors can prevent SIDS. Therefore, sleep movement monitors should not be routinely recommended by paediatricians. However, some of the studies have shown the potential for other uses for these monitors. There is some evidence to suggest that they may be of use for monitoring specific cohorts of infants, including those who have had a previous ALTE, or have cardiorespiratory risk factors. Further research into these areas is required.

\section{GP173 USAGE OF PROBIOTICS IN THE TREATMENT OF GASTROENTERITIS IN THE PAEDIATRIC POPULATION - A SYSTEMATIC REVIEW}

Allison Bell, Laken Boochoon, Craig Duffy, Jia Jun Foo, Sarah Koscic, Nnedimma Ozoani, Abidur Rahman, Darolyn Tan, Eleanor Molloy*. Department of Paediatrics, Tallaght University Hospital, Trinity College Dublin, Dublin, Ireland

\subsection{6/archdischild-2019-epa.234}

Background Acute gastroenteritis is defined as a sudden onset of diarrhoea which in itself is the passage of 3 or more loose stools in a 24 hour period, or the passage of one or more bloody stools in the presence or absence of abdominal pain, fever, nausea and vomiting. Acute gastroenteritis is self-limiting lasting no longer than 2 weeks, however, it is a major cause of morbidity and mortality worldwide. Current primary treatment modalities include restoration of the acid-base balance, correction of electrolyte disturbances, and oral rehydration therapies. The aforementioned therapeutic regimens help to decrease morbidity and mortality, however, it seldom has an impact on the duration of the infection and its symptoms. Probiotics are believed to help reduce both the duration and severity of symptoms of gastroenteritis, however strain specific efficacy and inter-strain comparison has not yet been established.

Objective The aim of this systematic review was to examine established original research to determine the efficacy of probiotics in treating acute gastroenteritis in the paediatric population. We examined the different strains of probiotics utilized and their associated outcomes in treating gastroenteritis.

Methods An Embase search was carried out with the help of a medical librarian. Two independent reviewers screened title and abstract, followed by full text review using the programme Covidence. All reviewer conflicts were resolved by a third party. Data was extracted from the included articles to determine correlation and effects of probiotics.

Results Of the 581 results obtained from the search, 11 studies were included for data extraction after applying the inclusion and exclusion criteria. Majority of the studies showed probiotics reduced duration of diarrhoea (8 of 11 studies), and a reduced duration of hospitalisation (6 of 11 studies). Notably, 2 papers reported adverse effects, such as fungaemia, in immunocompromised and ICU patients. The different strains of probiotics that were examined in the selected papers include Saccharomyces boulardii, Lactobacillus casei, and Lactobacillus acidophilus.

Conclusion Probiotics reduced the duration diarrhoea symptoms and hospitalization. Usage of probiotics was however accompanied by minimal side effects but not indicated in immunocompromised patients with gastroenteritis. However, further research needs to be conducted to determine the strain specific efficacy and dosage requirements for treatment of gastroenteritis using large scale double blinded randomized control trials.

\section{GP174 ALLERGY-FOCUSED HISTORY QUESTIONNAIRE AND ASSESSMENT OF GENOTYPE OF POLYMORPHIC MARKER RS182549 IN THE MCM6GENE ALLOW TO OPTIMIZE THE DIET FOR CHILDREN WITH INFLAMMATORY BOWEL DISEASES}

${ }^{1}$ Irina Gordeeva, ${ }^{1,2}$ Svetlana Makarova*, Kirill Savostyanov ${ }^{1}$, Leyla Namazova-Baranova ${ }^{2}$ Alexandr Pushkov 1, Dasha Golubova 1, Andrey Surkov 1, Oksana Ereshko 1, Maria Golubova ${ }^{1}$. ${ }^{1}$ Federal state autonomous institution National Medical Research Center for Children's Health Ministry of Health Russian Federation, Moscow, Russian Federation; ${ }^{2}$ Pirogov Russian National Research Medical University, Moscow, Russian Federation

\subsection{6/archdischild-2019-epa.235}

Children with inflammatory bowel disease (IBD) often receive a dairy-free diet during remission period without evidence, which adversely affects their nutritional status.

The aim Of the study was to optimize the approaches to prescribing diets for children with IBD

Materials and methods 180 children aged 1 to 17 years with IBD (90 patients with Crohn's disease and 90 - with ulcerative colitis) in clinical remission were included in this study. Testing of Lactase deficiency included Lactose Intolerance quick test (LIQT) and Real-time PCR, using fluorescent TaqMan probes for analyzing the genotype of polymorphic marker $r s 182549$ in the MCM6 gene. Allergy-focused history questionnaire, morphological study of biopsy specimens of the intestine with the counting of eosinophils, dairy products «open food challenge» (with fecal calprotectin assessment) were used for identifying of cow's milk allergy (CMA). 